\title{
Model Development of Illness Perception and Consequences in Breast Cancer Patients
}

\author{
L Hoseini, F Lotfi Kashani, S Akbari, ME Akbari, S Sarafraz Mehr*
}

\begin{abstract}
Breast cancer is the first or second malignancy among women worldwide. Illness perception (IP) and quality of life $(\mathrm{QoL})$ are major issues considering breast cancer management. An attempt was here made to inspect the predictive variables influencing IP and their impact on QoL in cancer patients. The key predictors adapted from previous studies including life satisfaction, perceived social support, self-esteem, hope, optimism, and spiritual well being were taken into account. Our sample included 200 female volunteers suffering from breast cancer applying exclusion criteria. The data were collected using various questionnaires and statistically analyzed by means of path analysis and structural equation modeling. The results revealed that of the six predictors, spiritual well being and social support had direct effects on QoL and IP. The only path with significant indirect correlation with IP was social support. Spiritual well being had the second significant direct effect on IP. Self-esteem occupied the third rank in direct effects on both QoL and IP. In conclusion, here in Iran and most likely in other strongly religious communities, spiritual intervention is an effective strategy to raise QoL. Also social support helps women suffering from breast cancer to experience better conception and coping strategies.
\end{abstract}

Keywords: Breast cancer - illness perception - quality of life - spirituality - Iran

Asian Pac J Cancer Prev, 17, Cancer Control in Western Asia Special Issue, 185-190

\section{Introduction}

Breast cancer is the second malignancy common among women throughout the world and is still the heading reason for women's untimely death over the last decades in some countries (Taghavi et al., 2012). As a result of improvements in early diagnosis, surgery and adjuvant therapies, the number of breast cancer deaths has significantly decreased and it turned into the most prevalent female cancer. In Iran, breast cancer is the first malignancy among both sexes with age standard rate (ASR) around 30 per 100,000 (Taghavi et al., 2012). Therefore, foremost psychosocial problems are highly evident during the diagnosis, treatment and follow up period albeit these problems can be reduced to some extent by psychological interventions (Carlsen, Jensen, Jacobsen, Krasnik, and Johansen, 2005). This has called for growing research interest on Quality of life (QoL) and the role of psychological factors affecting women's lives after diagnosis or treatment (Graves et al., 2007). QoL is an idea that has been used for the first time in oncology to cover up the practical outcomes of cancer and its management, as marked by the patients (Kaptein et al., 2011). Today, the QoL construction offers a general sense of well-being in multiple areas of life. On the other hand, the results of QoL research in patients suffering from cancer revealed a sense of capability in physical, emotional, social and spiritual dimensions of their lives (Härtl et al., 2010). More recent studies on behavioral phase of cancer, nevertheless, are inclined to go further than the concept of QoL. Recent theories and their clinical applications, psychological in nature yet practical in medicine, focus on self-regulation (Kaptein et al., 2011) . Therefore, some encouraging psychological approaches focus on patients' attitudes towards their illness (Stafford, Berk, and Jackson, 2009).The Self-Regulation Model (SRM), expanded by Leventhal (Leventhal, Brissette, and Leventhal, 2003), covered illness perceptions and coping strategies as determinants of QoL (Kaptein et al., 2011). Fundamentally, the SRM implies that people sort out their beliefs about illness around five elements (briefly introduced in the following paragraph), which have been termed Illness Perceptions (Aalto, Heijmans, Weinman, and Aro, 2005).

These components consist of thoughts about (a) identity, i.e. the symptoms the patient links with the illness, (b) cause, (c) consequences, (d) timeline, i.e. how long the patient considers the illness will last, and (e) cure/control . Although, the patient's beliefs somehow determine the QoL, rather slight work has explored their determinants in homogenous patient groups (Aalto et al., 2005). Moreover, while the self regulatory theory has accepted the fact that IP is affected by many factors, it has not been clearly established how IP is developed and how it might be 
modified (Aalto et al., 2005). Leventhal (Leventhal et al., 2003) has proposed that IP is affected by a range of personal, cultural and social factors. The significance of this study lies in the fact that, unlike previous ones, it clarifies the factors affecting both IP and QoL. Given that the patients' attitudes towards illness build up higher QoL, we aim to examine some predictable variables affecting IP as a median and QoL as a criterion for breast cancer patients. Since the mentioned studies were on the basis of other countries' patients and mainly on the vernacular population, they were not applicable for the Iranian patients.

We took into consideration the past important variables among other studies such as optimism, satisfaction with life, hope, self -esteem, spiritual well being, and perceived social support (Aalto et al., 2005; Butow et al., 2000; Foster et al., 2008; Frostholm et al., 2007; Härtl et al., 2010; Rifkin, Doddi, Karagji, and Pollack, 1999).

This model can be possibly applied as a pointer for preventing the frustrating perception of illness on the breast cancer patients in a practical manner for the women suffering from the illness in the region. The model is illustrated in Figure 1.

\section{Materials and Methods}

The present study was a non-experimental one, to be more precise, a correlation study. Considering our hypothesis, instead of regression, path analysis was employed to reach optimal results of the default model. Path analysis allows for model verification and establishment of the relation among variables. To address the main question put forward by the study, whether the specified model of QoL in breast cancer patients fits the experimental data or not, we have applied path analysis and Lisrel software version 8.7.

The population included 200 women ages between 25 and 65 and one case 18, suffering from breast cancer being treated by surgery, chemotherapy and radiation therapy, either separately or combined. The sampling in the present study was carried out selectively. To do this, among the women receiving treatment from different health centers, some were selected in non probability (non random) sampling during June 2012 till June 2013 and they all consciously agreed to sign the Consent Form.

Considering the goal of the research, the used instrument was a questionnaire. For assessing our six
Table 1. Demographic Characteristics of the Sample

\begin{tabular}{lccc}
\hline Age & Distribution & $\%$ & $\begin{array}{c}\text { Condensation } \\
\text { Distribution }\end{array}$ \\
\hline Under 20 & 1.0 & 0.6 & 0.6 \\
$21-30$ & 37.0 & 21.0 & 21.7 \\
$31-40$ & 41.0 & 23.3 & 45.0 \\
$41-50$ & 57.0 & 32.4 & 77.4 \\
$51-60$ & 36.0 & 20.5 & 97.9 \\
Over60 & 4.0 & 2.2 & 100.0 \\
Total & 175.0 & 100.0 & \\
Marital status & & & \\
Single & 37.0 & 21.0 & 21.0 \\
married & 139.0 & 79.0 & 100.0 \\
\hline
\end{tabular}

predictor variables (Satisfaction with life, optimism, spiritual well being, hope, perceived social support and self-esteem) plus one median variable (illness perception) and one criterion variable (QoL), we applied different appropriate questionnaires which will be described shortly afterwards. In finalizing the questionnaire, the classical measure analysis was conducted: field item analysis, reliability and validity tests. To determine the validity of the questionnaire, we investigated the face, content and construct validities. For instance, to find out the face and content validity of our instrument, we collected the viewpoints of some elites of the field until the researcher became sure of the reliability of the study. Meanwhile, the construct validity of the questionnaires has been investigated by the confirmatory factor analysis.

\section{Quality of life}

The EORTC QLQ-C23 (the European Organization for Research and Treatment of Cancer QoL Questionnaire) is a QoL questionnaire (Fayers and Bottomley, 2002) containing 23 questions, organized into scales consisting of an overall health standing scale, 5 functional scales, and 9 symptom scales. For the general health and functional scales, higher scores specify better general health and functioning. For the symptom scales, higher score is a sign of aggravated symptoms. The Iranian version of the EORTC QLQ-C23 was developed following precise EORTC translation process and has been validated for breast cancer (Montazeri et al., 2008).

\section{Illness Perception}

In the present study, we have chosen the Brief IP

Table 2. Descriptive Statistics Variables

\begin{tabular}{lccccc}
\hline Variables & Min & Max & Mean & Standard error s of mean & Standard deviation \\
\hline Illness perception & 1.4 & 9.4 & 6.1 & 0.1 & 1.6 \\
Satisfaction & 1.4 & 6.0 & 3.5 & 0.1 & 0.0 \\
Optimism & 1.6 & 5.8 & 3.1 & 0.1 & 0.7 \\
Hope & 1.4 & 4.4 & 2.9 & 0.1 & 0.6 \\
Social support & 1.0 & 5.4 & 2.6 & 0.1 & 1.0 \\
Self-esteem & 1.1 & 5.7 & 2.9 & 0.1 & 0.9 \\
Spirituality & 1.0 & 5.6 & 2.4 & 0.1 & 0.9 \\
QoL & 1.4 & 3.7 & 2.8 & 0.0 & 0.4 \\
\hline
\end{tabular}

186 Asian Pacific Journal of Cancer Prevention, Vol 17, Cancer Control in Western Asia Special Issue, 2016 
Table 3. Correlation Matrix of Variables

\begin{tabular}{|c|c|c|c|c|c|c|c|}
\hline & QoL & IP & Satisfaction & Optimism & Hope & Social support & Self esteem \\
\hline Illness perception & 0.3 & & & & & & \\
\hline Satisfaction & 0.2 & -0.3 & & & & & \\
\hline Optimism & 0.1 & -0.4 & 0.5 & & & & \\
\hline Hope & 0.2 & -0.4 & 0.5 & 0.6 & & & \\
\hline S. support & 0.1 & -0.1 & 0.4 & 0.2 & 0.2 & & \\
\hline Self-esteem & 0.2 & -0.3 & 0.6 & 0.4 & 0.6 & 0.2 & \\
\hline Spirituality & 0.2 & -0.4 & 0.6 & 0.5 & 0.7 & 0.4 & 0.6 \\
\hline
\end{tabular}

Questionnaire. The Brief IPQ has nine items. Therefore, it contains eight new items plus part of the causal scale previously used in the IPQ-R. All items except the essay-type questions are rated using a 0 -to-10 response scale. Five of the items assess cognitive illness representations. Two of the items assess emotional representations. One item assesses illness clarity. Assessment of the causal representation is by an open-ended response item adapted from the IPQ-R, which asks patients to list the three most important causal factors in (Figueiras and Alves, 2007; Kalantari et al., 2012; Sampaio, Pereira, and Winck, 2012) Optimism: It means the score a subject in the study gets from the 6 question optimism oriented questionnaire of Carver (Carver et al., 1993). This questionnaire is also known as Life Orientation Test (LOT). Hope: The hope assessment tool is the adult dispositional hope scale in adults aged 15 years and above. It is a self report questionnaire that contains twelve questions raised by Snyder (Snyder et al., 1991) and its psychological features have been approved. Items are scored on an eight point Likert scale. Total hope scale scores range from a minimum of 8 to a maximum of 64, with high scores reflecting high levels of hope (Snyder et al., 1991). Perceived Social Support: To assess this variable we applied the Multidimensional Scale of Perceived Social Support of Zimet (Zimet, Dahlem, Zimet, and Farley, 1988), which contains three subscales-family, friends, and other significant support. This scale includes 12 questions for assessing 3 subscales.

\section{Satisfaction with Life}

For assessing satisfaction with life, satisfaction with life scale has been used, raised and developed by Diener (Diener, Emmons, Larsen, and Griffin, 1985). It includes 3 factors; life satisfaction, negative and positive effect. These factors were revealed in 5 items on the basis of "semantic similarity" (Diener et al., 1985). Respondents are told to rate each item using a 7-point scale ranging from 1 (strongly disagree) to 7 (strongly agree). Item ratings are summed up to provide a total score ranging from 5 - 35 where higher scores are indicative of greater life satisfaction. (Diener et al., 1985; Pavot and Diener, 1993).

\section{Self-esteem}

Rosenberg Self-Esteem Scale is a ten item Likert scale with items answered on a four point scale - from strongly agree to strongly disagree (Rosenberg, Rosenberg, and McCord, 1978).

\section{Spiritual well being}

The Spiritual well being scale (SWBS) developed by Paloutzian is one of foremost applied tools in psychology. The 20 item scale is composed of two subscales which contains religious well-being subscale and the existential well-being subscale.

In the cases in which the patient was illiterate, was not able to read without wearing glasses or was not willing to read and answer the questionnaire thoroughly, the researcher had to read the questions and mark the answers by herself. On the whole, 176 questioners were reliable enough to be used in the study. It is noteworthy that before analysing any data, we made sure of the accuracy of the filled questionnaire by data screening in an attempt to reach this significant goal: Eliminating patients who left out more than $20 \%$ of the questions blank and eliminating patients who had the parallel answer patterns as well. The analyses were executed on 176 valid questionnaires. For the next step, we improved or removed outlier data. From the hypothesis of parametric data gathering, normal and homogenious nature was studied. Of course, since almost all of the data is in Likert scale and from the theoretical view is in ordinal scale, we took them into consideration. The final step in data screening has been replacing blank left out questions and to do this

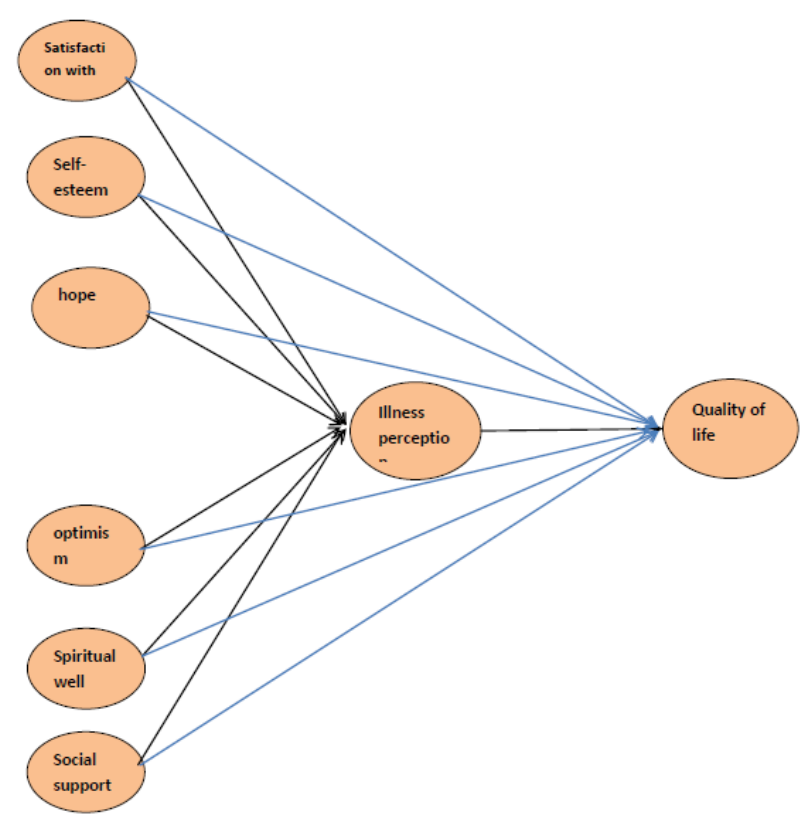

Figure 1. Path diagram: A Pictorial Representation of the Model 
Table 4. The Fit Indices of the Model

\begin{tabular}{lllllllll}
\hline AGFI & GFI & CFI & NNFI & NFI & RMSEA & df $/ 2 \chi$ & df & Fit indices \\
\hline 0.9 & 0.9 & 1.0 & 0.1 & 1.0 & 0.0 & 1.2 & 3.0 & Initial amount \\
0.9 & 1.0 & 1.0 & 1.0 & 1.0 & 0.0 & 1.7 & 3.0 & Modified amount \\
more than0.9 & more than0.9 & more than0.9 & more than 0.9 & more than 0.9 & less than0.1 & less than 0.1 & 3.0 & Acceptable amount \\
\hline
\end{tabular}

we utilized Expectation-Maximization (EM) method.

\section{Results}

The characteristics of the study samples are shown in Table 1. Moreover, $49.4 \%$ of them consisted of housewives and $50.6 \%$ were working women. As can be seen in Table 2, from the predictor variables the prominent mean is satisfaction with life and the lowest mean is spiritual well-being. The results of path analysis showed degrees of correlation between the variables (Table 3).

The results in table 3 show that in 95\% level, there is a meaningful correlation between all variables except optimism and social support with the QoL, and social support with IP $(p<0.05)$. The initial founding data for these indices show unacceptable model fit, so, the model has been revised and the modifications are meaningful. In Table 3 the most important indices of fitting test of the conceptual model is summarized.

Regarding that each of the presented indices is not merely enough for passing judgment on the fitness or unfitness of the model, it is essential that these indices be juxtaposed. Table 3 shows that after applying the necessary improvements, the fit model indices have been modified in an appropriate state, and as a result the research hypothesis is hereby answered. For the next step in the final (modified) model, the standard coefficient path $(\beta)$ is reported.

Path coefficient test in Figure 2 shows that the whole standard path coefficient (the direct effects of all predictors on IPand QoL) was significant in 95\% level, except for the direct effect of optimism and hope on IP and perceived social support on QoL as well. $(\mathrm{p}<0.05)$ moreover, in order to modify the final model, the direct path from hope to QoL and satisfaction with life to IP were eliminated; so that no amount of $\beta$ is acquired for those two paths. Another important issue that the present study is trying to address is to estimate the indirect effect of predictor on QoL with a mediation of IP as a median variable. The results of indirect effect testing by Lisrel software showed that only indirect effect of perceived social support on

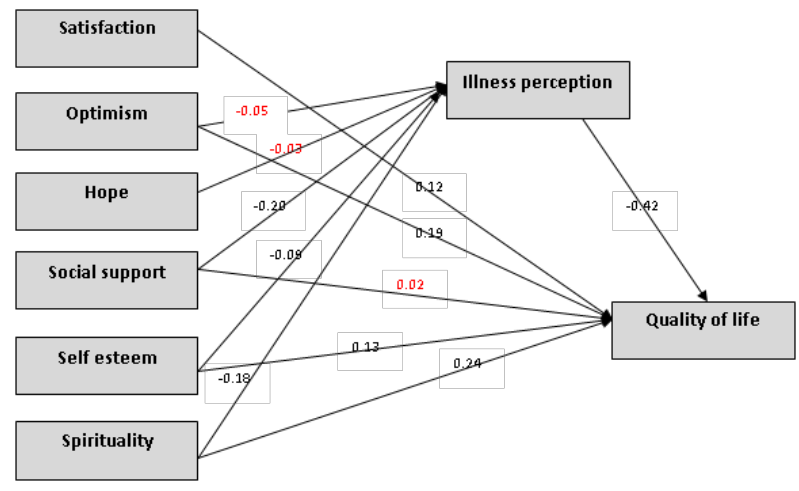

Figure 2. Standardized Path Coefficients of the Modified Model
QoL with the mediation of IP is significant $(\beta=0.1)$ and the indirect effect of other predictors are not significant on QoL ( $p>0.05$ ). Also most of the variables are significant. In order to indicate a "large" direct effect or a "small" one, we employed significance tests. The result of tests reflects the absolute magnitudes of path coefficients.

\section{Discussion}

Based on World Health Organization regulation (Sulmasy, 2002) the aspects for comprehensive health are biological, psychological, social and spiritual dimensions. The spiritual aspect has been the center of attention through the science history and more attention in Abrahamic religions such as Judism, Christianity, and Islam. It is well accepted that spiritual health affects the quality of life and even the life expectancy and quantity of life. Also in our study the results revealed among the six predictors spiritual well being has the highest direct effect on QoL in Iranian women and the second significant effect on IP. A possible explanation for that is spirituality helps people to rely on a superior force to fight against the negative effects of stress and to better manage the disease. It also directs people to go beyond the corporeality of life to find meaningful aspiration for their life. Considering the religious context (Iranian people deeply believe in the spiritual dimension of life), it will ensure a higher impact on QoL. This result is consistent with other past studies. Spirituality has been an important issue in the last decade which has attracted the attention of mental health professionals. Based on some previous studies, spirituality is a strong predictor of mental health and well being through which social support influences health by regulating the persons' evaluation of the tenseness of the situation. Social support has been related to perception of stress (Helgeson and Cohen, 1996). A chronic illness can be seen as a source of stress caused by its adaptation difficulties (Fallah, Golzari, Dastani, and Akbari, 2011; Vermandere et al., 2011). When people believe that there is a source of power and energy that loves them on which they can rely, they find a higher sense of control over events and feel less fear and apprehension of the consequences of disease. They come along with the time line of illness more easily, and experience fewer negative thoughts and emotions (Rifkin et al., 1999). Meraviglia found that some cancer patients confess that spirituality can increase withstanding the physical and psychological crisis that followed the diagnosis and treatment of cancer (Meraviglia, 2006). Moreover, it has been observed in women with breast cancer who seek spirituality, it can be viewed as an effective source for coping with negative physical and psychological responses to breast cancer. The perceived social support has a direct effect on IP and also the highest indirect effect on QoL. To explain

188 Asian Pacific Journal of Cancer Prevention, Vol 17, Cancer Control in Western Asia Special Issue, 2016 
this, we might point out the theory of social support, which suggest that one possible way for sufferers is evaluation of illness affected by support received from others. This result is also consistent with other research; Helgeson and Cohen (1996) showed among cancer patients receiving social support, it acts as a shield against the negative consequences of cancer treatment. One of the key roles of the family and the medical team is to create a supportive environment. Supportive measures improve coping strategies people use to deal with stress which affect health and QoL by protecting the individuals against the negative consequences of stress. Self-esteem has third rank in both direct effect on QoL and IP. One explanation for the significant relationship between selfesteem and IP is that people with high self-esteem, feel greater control over their disease process and treatment and at diagnosis they are less prone to panic. Cohen and Lazarus (1983) showed people with high self esteem believe that they have the capabilities necessary to meet the needs of a threatening situation and thus turn it to a challenge. Also about the relation between self-esteem and QoL, Brinden et al (2002), showed people who have lower self-esteem, willingness to set goals are also less likely to achieve objectives. They are elusive from communicating with others and have more fears of their audience and ultimately they will have weaker self-concept. In conflict people with high self-esteem is more receptive and honest and can easily communicate with others to establish the better relation. This relationship will serve to reinforce their positive self concept and help to raise the QoL. The fourth order belongs to optimism on direct effect of QoL and after that satisfaction with life plays its role. Optimism is the point of view that good things are likely to happen. Optimists are liable to experience life's difficulties with less misery than do pessimists (Scheier and Carver, 1992). They are also likely to have better health habits, better mental and physical health, and faster curing when they become sick in comparison with pessimists. Psychology's ultimate goal is to improve the quality of human life through the processes of human cognition and behavior. People who have high sense of satisfaction with life feel much more fun and enjoy their lives (Diener, Oishi, and Lucas, 2003). Life satisfaction is remarkable from an emotional assessment view in that it is more cognitively than emotionally driven. Life satisfaction can affect all-purpose region of life. People who are satisfied with life are more patient and feel more relaxed in facing and coping with health problems and they are better at dealing with health difficulties (Diener et al., 2003). No significant direct effects of optimism and hope with IP have been seen. Although, Optimism is often associated with better immune functioning and optimists keep on in tough labors to control stressful conditions (Roy et al., 2010). Some research found some controversial results. Weinstein (Weinstein et al., 2003) studied how optimistic people view their future health by asking them, some questions. These results termed as unrealistically optimistic, this optimism is based on illogical ideas-for instance, some people think they are at lower risk than other people if the health problem happens rarely. Unrealistic optimism causes over-optimism in many individuals, leading them to think that protection is not so necessary anymore. The direct path from hope to QoL was omitted in order to modify the model. The linear relation with the IP and the QoL was significant. These results are compatible with previous studies and QoL has the most powerful effect on QoL between the first 6 predictors. Recent studies of Petri showed that IP is associated with selfmanagement behaviors and QoL in chronic disease (Petrie, Jago, and Devcich, 2007) Kapti et al (2011) reveal IP in Huntington patients strongly affects the physical and mental well-being. Ponz et al (2006) showed in Diabetes type II patients' perception of short time line and control of illness has a positive effect on self management behavior. Scharloo et al (2005) revealed the significant relation between IP and QoL in different kind of cancer patients.

Although, nowadays more studies are designed to focus on illness perception behavioral and emotional outcomes; little work has been done to investigate impact factors of those components. The new area for researchers is to identify and introduce important factors which are related or affected illness perception and also are important for interventions and those consequential outcomes such as quality of life. In future, interventions should be designed to target the most important personal, cultural and social factors which shape IP. Our model has the capability to specify key factors effect on IP and QoL. As a result we can apply it as guidance on how preventive interventions should target these factors or more others to influenced IP and QoL after that. In recent decades spirituality and spiritual care is validated in some countries. Here in Iran it can be referred to specialists as a powerful tool. In addition, social support always has been on top of consideration and has proven its advantage. More research is needed to identify the accuracy of the model, and whether other else factors are affected.

Conclusion: Managing the breast cancer patients in an established manner needs to be more comprehensive for health care regarding more attention to spiritual, social and psychological aspects to get better outcome for quality and quantity of life for the patients and care givers.

\section{References}

Aalto AM, Heijmans M, Weinman J, Aro AR (2005). Illness perceptions in coronary heart disease: Sociodemographic, illness-related, and psychosocial correlates. J Psychosom Res, 58, 393-402.

Brinton L, Lacey J, Devesa S (2002). Epidemiology of breast cancer. Cancer of the Breast, 5, 111-32.

Butow PN, Hiller JE, Price MA, et al (2000). Epidemiological evidence for a relationship between life events, coping style, and personality factors in the development of breast cancer. J Psychosom Res, 49, 169-81.

Carlsen K, Jensen AB, Jacobsen E, Krasnik M, Johansen C (2005). Psychosocial aspects of lung cancer. Lung cancer, 47, 293-300.

Carver CS, Pozo C, Harris SD, et al (1993). How coping mediates the effect of optimism on distress: a study of women with early stage breast cancer. J Pers Soc Psychol, 65, 375-401.

Cohen F, Lazarus RS (1983). Coping and adaptation in health and illness. Handbook of health, health care, and the health professions, 608-635. 
Diener E, Emmons RA, Larsen RJ, Griffin S (1985). The satisfaction with life scale. J Pers Assess, 49, 71-5.

Diener E, Oishi S, Lucas RE (2003). Personality, culture, and subjective well-being: Emotional and cognitive evaluations of life. Annu Rev Psychol, 54, 403-25.

Fallah R, Golzari M, Dastani M, Akbari M (2011). Integrating spirituality into a group psychotherapy program for women surviving from breast cancer. Iran J Cancer Prev, 4, 141-7.

Fayers P, Bottomley AO (2002). Quality of life research within the EORTC-the EORTC QLQ-C30. Eur J Cancer, 38, 125-33.

Figueiras MJ, Alves NC (2007). Lay perceptions of serious illnesses: An adapted version of the Revised Illness Perception Questionnaire (IPQ-R) for healthy people. Health Psychol, 22, 143-58.

Foster NE, Bishop A, Thomas E, et al (2008). Illness perceptions of low back pain patients in primary care: what are they, do they change and are they associated with outcome. Pain, 136, $177-87$.

Frostholm L, Oernboel E, Christensen KS, et al (2007). Do illness perceptions predict health outcomes in primary care patients? A 2-year follow-up study. J Psychosom Res, 62, 129-38.

Graves KD, Arnold SM, Love CL, et al (2007). Distress screening in a multidisciplinary lung cancer clinic: prevalence and predictors of clinically significant distress. Lung cancer, 55, 215-24.

Härtl K, Schennach R, Müller M, et al (2010). Quality of life, anxiety, and oncological factors: a follow-up study of breast cancer patients. Psychosom Med, 51, 112-23.

Helgeson VS, Cohen S (1996). Social support and adjustment to cancer: reconciling descriptive, correlational, and intervention research. J Health Psychol, 15, 135-38.

Kalantari H, Sararoodi RB, Afshar H, et al (2012). Relationship between illness perceptions and quality of life in patients with irritable bowel syndrome. J Mazandaran Univ Med Sci, 22, 145-49.

Kaptein AA, Yamaoka K, Snoei L, et al (2011). Illness perceptions and quality of life in Japanese and Dutch patients with non-small-cell lung cancer. Lung cancer, 72, 384-90.

Leventhal H, Brissette I, Leventhal EA(2003). The common-sense model of self-regulation of health and illness. The self-regulation of health and illness behaviour, 1, 42-65.

Meraviglia M (2006). Effects of spirituality in breast cancer survivors. Oncol Nurs Forum, 33, 1-7.

Montazeri A, Vahdaninia M, Harirchi I, et al (2008). Quality of life in patients with breast cancer before and after diagnosis: an eighteen months follow-up study. BMC cancer, $\mathbf{8}, 330$.

Pavot W, Diener E (1993). Review of the satisfaction with life scale. Psychol Assessment, 5, 164-72.

Petrie KJ, Jago LA, Devcich DA (2007). The role of illness perceptions in patients with medical conditions. Curr Opin Psychiatry, 20, 163-67.

Ponzo MG, Gucciardi E, Weiland M, et al (2006). Gender, ethnocultural, and psychosocial barriers to diabetes self-management in Italian women and men with type 2 diabetes. J Behav Med, 31, 153-60.

Rifkin A, Doddi S, Karagji B, Pollack S (1999). Religious and other predictors of psychosocial adjustment in cancer patients. Psychosomatics, 40, 251-6.

Rosenberg FR, Rosenberg M, McCord J (1978). Self-esteem and delinquency. $J$ Youth Adolesc, 7, 279-94.

Roy B, Diez-Roux AV, Seeman T, et al (2010). Association of optimism and pessimism with inflammation and hemostasis in the Multi-Ethnic Study of Atherosclerosis (MESA). Psychosom Med, 72, 134-40.

Sampaio R, Pereira MG, Winck JC (2012). Psychological morbidity, illness representations, and quality of life in female and male patients with obstructive sleep apnea syndrome. Psychol Health Med, 17, 136-49.

Scharloo M, de Jong B, Robert J, et al (2005). Quality of life and illness perceptions in patients with recently diagnosed head and neck cancer. Head neck, 27, 857-63.

Scheier MF, Carver CS (1992). Effects of optimism on psychological and physical well-being: Theoretical overview and empirical update. Cog Therapy Res, 16, 201-28.

Snyder CR, Harris C, Anderson JR, et al (1991). The will and the ways: development and validation of an individual differences measure of hope. J Pers Soc Psychol, 60, 570-85.

Stafford L, Berk M, Jackson HJ (2009). Are illness perceptions about coronary artery disease predictive of depression and quality of life outcomes?. J Psychosom Res, 66, 211-20.

Sulmasy DP (2002). A biopsychosocial-spiritual model for the care of patients at the end of life. Gerontologist, 42, 24-33.

Taghavi A, Fazeli Z, Vahedi M, et al (2012). Increased trend of breast cancer mortality in Iran. Asian Pac J Cancer Prev, 13, 367-70.

Vermandere M, De Lepeleire J, Smeets L, et al (2011). Spirituality in general practice: a qualitative evidence synthesis. Br J Gen Pract, 61, 749-60.

Weinstein MC, O’Brien B, Hornberger J, et al (2003). Principles of good practice for decision analytic modeling in health-care evaluation: Report of the ISPOR task force on good research practices-modeling studies. Value Health Reg, 6, 9-17.

Zimet GD, Dahlem NW, Zimet SG, Farley GK (1988). The multidimensional scale of perceived social support. J Pers Asses, 52, 30-41. 